\title{
Image Quality Control for Digital Mammographic Systems: Initial Experience and Outlook
}

\author{
Hans Roehrig, Tong Yu, and Elizabeth Krupinski
}

\begin{abstract}
This report presents (1) a broad topical review and a tutorial of the possibilities for image quality control (10C) with digital systems, and (2) results and initial experience for IOC with two commercial digital imaging systems, but with limited discussion on any particular method. Digital imaging systems used for mammographically guided digital stereotactic breast biopsy were evaluated extensively at the University of Arizona. Measurements were made of linearity, sensitivity, signal-to-noise ratio, and square-wave modulation. Images of phantoms such as the American College of Radiology Accreditation Phantom and the contrast detail mammography Phantom were evaluated as well as images of the x-ray source's focal spot. The evaluation also included the cathode ray tubes for the imaging systems. The data collected show that digital imaging systems have an important advantage over film-screen systems because they provide a digital signal as output that can be used for quantitative analysis. As a result, IOC can become a much more quantitative discipline than presently practiced, providing more information on the imaging systems under evaluation, and providing better control over their properties during actual operation.

Copyright 11995 by W.B. Saunders Company
\end{abstract}

KEY WORDS: image quality control (IOC), digital mammography, quantitative image analysis, contrast detail studies.

$\mathbf{I}^{\mathrm{M}}$ MAGE QUALITY CONTROL (IQC) is playing an increasingly important role in the diagnostic radiology department. It has been shown that a successful IQC program can achieve three goals': (1) reduced dose to the patient, (2) improved diagnosis, and (3) savings in expenses. Note the correlation of the three "D"s: dose, diagnosis and dollars! This is particularly true after the successful introduction of digital imaging modalities such as digital subtraction angiography, computerized tomog-

From the Department of Radiology, Arizona Health Sciences Center, University of Arizona at Tucson.

Supported by National Institutes of Health (NIH) Grant No. 1 R29 CA56073-01 and by NIH Grant No. 1 R 43 CA56249-01.

Address reprint requests to Hans Roehrig, PhD, Department of Radiology, Arizona Health Sciences Center, University of Arizona, Tucson, AZ 85724.

Copyright $₫ 1995$ by W.B. Saunders Company

0897-1889/95/0802-0005\$3.0010 raphy, computed radiography, and magnetic resonance imaging, where accuracy is of highest importance. Now it is virtually impossible to run a radiology department without a credible IQC program.

Digital mammography has arrived in the radiology department to a certain degree with the introduction of systems used for mammographically guided digital stereotactic breast biopsy. ${ }^{2,3}$ Image quality is particularly important here because it is the radiologic modality that requires the highest spatial and contrast resolution. ${ }^{4}$ Additionally, the size of digital mammograms is expected to be at least $2,048 \times 2,560$ pixels $\times 12$ bits, ${ }^{5}$ making IQC even more critical.

IQC is already the cornerstone of practicing high-quality mammography. To ensure high quality, The American College of Radiology (ACR) has established a voluntary program for the accreditation of mammographic screening sites. ${ }^{6-10}$ One reason for this is that a recent survey among various film-screen mammography centers showed large differences in film densities, patient dose, and objects detected in the ACR phantom. ${ }^{7,8}$ A major reason for these differences is large variations in techniques, as well as large differences in film-processor properties. It is concluded that there is a significant lack of standardized IQC practices in the United States.

The Federal Government has also recognized the importance of image quality in mammography. The 1992 Mammography Quality Standards Act set strict new standards and requirements. ${ }^{11}$ MQSA regulations now being written will indirectly affect other radiology and imaging activities. To regulate imaging equipment, personnel and facilities, the Department of Health and Human Services just created a Division of Mammography Quality and Radiation Programs within the Food and Drug Administration.

This communication is intended to be a broad topical review and tutorial as well as a presentation of results and initial experience for IQC 
with two commercial digital imaging systems used in mammographic applications. As a result, there is no in depth discussion of any particular method.

\section{IQC WITH CONVENTIONAL FILM-SCREEN SYSTEMS}

IQC for the conventional film-screen combination concentrates on evaluating the optical density and the contrast (density difference) of the developed film of the film-screen combination. ${ }^{1,7-10}$ Specific steps are (1) exposure of the film to a range of photon fluences from a light source (simulating the mostly visible light emission of the phosphor screen in the form of a "step wedge"), (2) exposure of the film-screen combination to a particular $\mathrm{x}$-ray fluence, and (3) analysis of the optical density to which the film has been developed. Most commonly, the preferred optical density (OD) in the object area of interest is $\sim 1.2$. The analysis of the OD is done mostly with the aid of a densitometer, assuring that the density for the same photon fluence is always the same, and assuring that the contrast, the difference in the OD between certain steps in the step wedge is always the same. Note that these tests do include problems with the film developer. In fact, in most cases, the cause of insufficient image quality in the developed film is the film developer.

IQC also covers spatial resolution by determining the "limiting spatial resolution". Here an x-ray image of a lead-bar spatial resolution pattern is generated, providing an image with spatial frequencies in the range from very low $(0.05 \mathrm{lp} / \mathrm{mm})$ to very high spatial frequencies $(>20 \mathrm{lp} / \mathrm{mm})$. An observer determines the highest spatial frequency (finest pattern) he can barely see. Of particular importance to spatial resolution is proper film-screen contact. Film screen contact is checked by imaging a very fine grid.

IQC with film-screen systems also includes inspection of the x-ray source. Most commonly, one determines (1) the exposure in the plane of the imaging systems for a given kilovolt peak and milliamperes setting ( $\mathrm{mR} / \mathrm{mAs})$, (2) the effective energy in terms of half-value layer of aluminum $(\mathrm{HVL})_{\mathrm{A} 1}$ necessary to reduce the beam intensity by a factor of two ("the poor man's x-ray spectrum"), and (3) the size of the focal spot. ${ }^{12}$ Determination of the actual x-ray spectrum, which among others would provide a precise measure of the actual generator voltage, is rarely done as only few laboratories have the specialized equipment necessary (like a $\mathrm{Ge}$ spectrometer). ${ }^{13-16}$ However, such measurement would be extremely important as it has been shown that changes in $\mathrm{x}$-ray source voltage by less than $1 \mathrm{kV}$ will affect image quality, ie, density as well as contrast. ${ }^{17}$

Unfortunately, two of the most important performance parameters of imaging systems, noise and signal-to-noise ratio, are rarely included in IQC programs for film-screen systems because the procedure for measuring film noise is very involved. ${ }^{18,19}$ The common method to measure film noise is to use a microdensitometer and to sample the OD of the film at different closely spaced positions within the image ("scanning") with an aperture of known diameter. Ideally the aperture diameter should match the diameter of an object of interest in mammography such as a microcalcification or a mass. ${ }^{20,21}$ A measure of the noise is the SD (root mean square; RMS) of the density for the samples taken.

Nevertheless, attempts are made to estimate the signal-to-noise ratio in film-screen systems. The most common approach is to use a contrastdetail phantom and to perform a contrast-detail study. ${ }^{22}$ A contrast-detail phantom is a device that contains a series of simple objects on a uniform background, like discs of different diameters (detail) and different thickness (contrast). Such a contrast-detail phantom is imaged at different exposure conditions (milliamperes and kilovolt peaks) and the images are displayed to human observers. ${ }^{22}$ The human observer identifies the objects that he can barely see (threshold contrast, $50 \%$ probability of detection). Such contrast-detail studies can be very quantitative. They permit estimating the display signal-tonoise ratio of a system for an object of a particular diameter ${ }^{21}$ However, the accuracy of such contrast-detail studies is limited because of large variations between observers. The accuracy can be increased by increasing the number of observers, making a contrast-detail study fairly costly in terms of resources. 
Several phantoms have been developed specifically for use in mammography, but not all phantoms offered for IQC in film-screen mammography are suited for contrast-detail studies.

The ACR accreditation phantom was developed by the ACR for use as an integral part of a mammographic accreditation program. ${ }^{6-8}$ The phantom contains test objects to simulate indicators of breast cancer. There are six nylon fibrils, simulating soft-tissue fibrils; five groups of six aluminum oxide specks, simulating clusters of microcalcifications; and there are five spheres simulating masses. Unfortunately this phantom cannot be used for contrast-detail experiments, because there are no provisions to determine the probability of detection.

The contrast detail mammography (CDMAM) phantom was developed at the University of Nijmegen, The Netherlands. It is a contrastdetail phantom that permits determination of threshold contrast as a function of object diameter. ${ }^{23}$ The results are plotted as a contrastdetail curve, presenting the observed threshold contrast for a particular x-ray exposure as a function of object size. The phantom consists of 16 rows and 16 columns spread over the $8-x$ 10 -inch format. The center-to-center distance of the rows and columns is $\sim 1 \mathrm{~cm}$, providing squares of approximately $1 \times 1 \mathrm{~cm}$. Each square contains two identical gold disks as objects to be detected. Each pair has a certain diameter and a certain thickness. One disk of the pair is in the center of the square and the other is in one of the four corners, randomly selected. Within a row, the disk-diameter is constant, and the thickness increases logarithmically from square to square. Within a column, the disk thickness is constant and the disk diameter increases logarithmically. The disk diameters range from 0.1 to $3.2 \mathrm{~mm}$, whereas the disk thickness varies from 0.05 to $1.6 \mu \mathrm{m}$.

Note that the traditional IQC for film-screen combinations is not very quantitative with respect to image quality. Particularly, with respect to display signal-to-noise ratio and spatial resolution: (1) it does not provide a measure of the display signal-to-noise ratio, which would be required to permit estimation of what object can be detected with which probability of detection; and (2) it only provides limiting resolution as a measure of spatial resolution, it does not provide modulation at different spatial frequencies.

\section{DIGITAL IMAGING SYSTEMS}

\section{General}

Digital imaging systems have many advantages over analog film-based systems because they provide a separation between the important functions of detection and display. ${ }^{24-27}$ However, this advantage is associated with a disadvantage, certainly in view of IQC: Image brightness on the CRT monitor (the electronic viewing medium for many digital imaging systems and the replacement for the film lightbox) is not necessarily a measure of $x$-ray exposure. Contrary to film, where overexposure and underexposure are clearly related to OD (and can be easily and reliably measured with the aid of a densitometer), the image brightness on the CRT of a digital system can be independent of the exposure to the $\mathrm{x}$-ray sensor. Recall that most CRTs have contrast and brightness controls, which permit setting contrast and brightness to a large range of values, independent of the actual video signal going into the CRT. Therefore IQC for digital imaging systems will have to resort to measures other than display brightness and display contrast. In fact, IQC will also have to include the display monitor (see below).

On the other hand, digital imaging systems have an important advantage over film-screen systems: they provide a digital signal as output, which is available almost instantaneously and can be used for quantitative analysis. This output can be digital numbers in the range of 0 to 255 digital units (analog-to-digital converter units [ADU] or gray levels [GL]) for an 8-bit system, or 0 to 4,096 ADU for a 12-bit system. Having a digital output will permit expanding IQC to a more quantitative discipline, providing more information on the imaging systems under evaluation, and providing better control over their properties during the actual operation.

\section{Digital Imaging Systems for Mammographic Applications}

At the University of Arizona, we have been using digital $\mathrm{x}$-ray imaging systems for mammographically guided stereotactic breast needle biopsies for some time. We first experimented 
with a system built in-house, based on an experimental charge coupled device (CCD) camera, using a phosphor screen, a 2:1 fiber-optic taper and a 1,024- $\times$ 1,024-pixel CCD, cooled and read out to permit 12-bit digitization. Later, two commercial systems were installed: system one, using a MinR-type screen (Eastman Kodak, Rochester, NY), a 2:1 fiber-optic taper and a $1,024 \times 1,024$-pixel CCD with 12-bit digitization; and system two, using a Lanex screen (Eastman Kodak), coupled by a lens to a 1,024 $\times$ 1,024-pixel CCD, also with 12-bit digitization., ${ }^{2,3}$ These systems have been used successfully to perform stereotactic breast needle biopsies and preoperative needle localizations. They turned out to be an extremely valuable tool for the biopsy procedures, reducing the procedure time from 45 to 20 minutes on average. Figure 1 shows schematics of both commercial systems.

Both digital imaging systems routinely use "flat fielding," a procedure that subtracts the offset caused by dark signals and bias and corrects ("normalizes") the raw image for signal

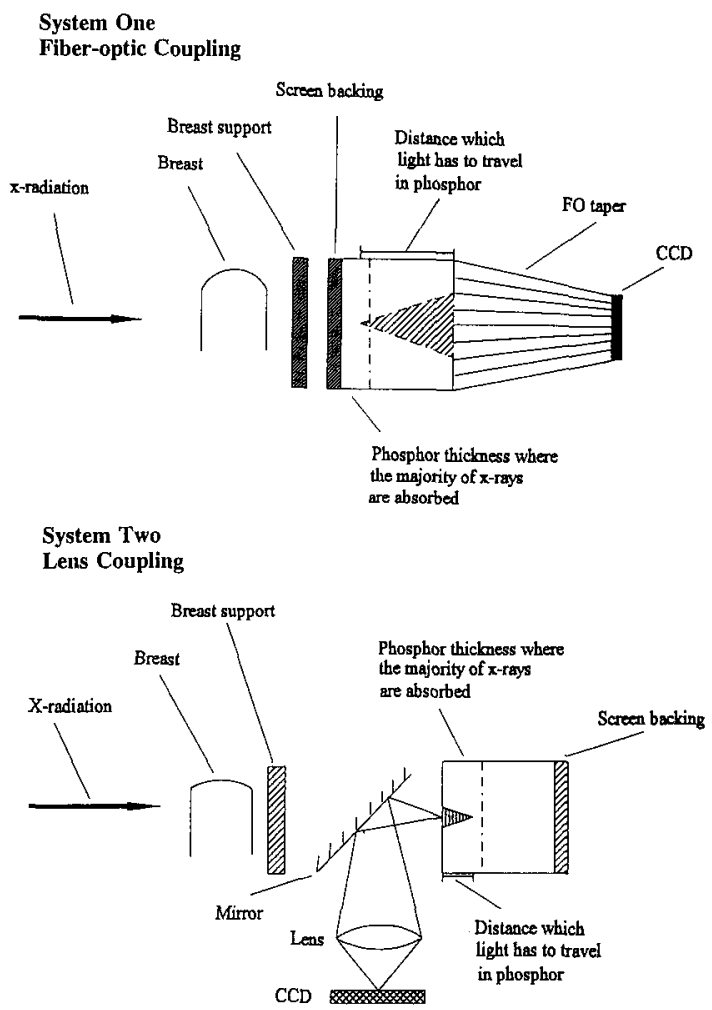

Fig 1. Schematics of system one (above) and system two (below), which are digital imaging systems used for mammographically guided stereotactic breast biopsy (reprinted with permission from Roehrig et a ${ }^{3}$ ). nonuniformities caused by local variations in CCD sensitivities, the $\cos ^{4}$ losses of a lens, the chicken-wire pattern of a fiber-optic taper or the heel effect of the $\mathrm{x}$-ray source..$^{28}$ In fact, this flat fielding process is what makes digital imaging systems superior to film-screen combinations as far as signal-to-noise ratio is concerned.

These digital systems were extensively evaluated at the University of Arizona., ${ }^{2,3}$ The results offered valuable insight into problems as well as prospects of IQC in digital mammography. In the following, the initial experience of the University of Arizona with IQC of digital imaging systems will be discussed in two parts: (1) IQC parameters useful for digital imaging systems and (2) variations of performance parameters as a function of time.

\section{IOC FOR DIGITAL IMAGING SYSTEMS}

\section{Performance Parameters Useful for Quantitative IQC}

It is to be emphasized that no recommendations are made here with regard to $x$-ray energy, other than to state that one should use a beam quality representative of the $\mathrm{x}$-rays emerging from the patient's breast. Referring to presently used exposure techniques, such a beam can be generated with a setting of $28 \mathrm{kVp}$ and a filtration of $4 \mathrm{~cm}$ of plexiglass. Such a beam might not be optimum for operation of digital mammography systems. Recall that the effective x-ray energy of conventional film-screen mammography is typically about $20 \mathrm{keV}$, which is useful for film-screen combinations because it provides good x-ray contrast. However, Fahrig et $a^{29}$ has shown that the optimum $x$-ray energy should be selected for optimum signal-to-noise ratio at a given glandular dose. To meet this criterion, the effective $\mathrm{x}$-ray energy should be between 25 and $28 \mathrm{keV}$ (depending on the thickness of the breast).

\section{Linearity and Sensitivity}

Many x-ray systems, like conventional film, have an output that is proportional to the log of the $\mathrm{x}$-ray exposure. However, the digital systems used for stereotactic applications are linear. Linearity is a system property that is desirable if certain image manipulations are to be performed. For instance, the modulation transfer function (MTF) of the imaging system can only 
be obtained from the Fourier Transform of the Line Spread Function, if the imaging system is linear. ${ }^{18}$ If the system is not linear, the data have to be linearized. It is for this reason that system linearity is of interest. Linearity of a digital system is found from a plot of the digital signal (the mean digital level in units of ADU in a region of interest formed by $100 \times 100$ pixels) as a function of the $x$-ray exposure to the image detector in milliroentgens. The system is linear if the slope of this plot on double-log scale is unity. When plotted linearly, the graph should be a straight line and go through the origin. Figure 2 shows examples of characteristic curves for systems one and two (solid graphs, left ordinate, using units of ADU, versus abscissa in milliroentgens) in comparison with that of filmscreen with and without a scatter grid (dashed graphs, right ordinate, using units of $O D$, versus abscissa in milliroentgens). Note again, the ordinate on the left side with units of ADU presenting the response of the digital systems, and the ordinate on the right side with units of OD presenting the response of the film-screen system. Both digital systems are indeed linear, whereas the film-screen system is nonlinear. The effective energy of the $x$-ray beam was about $18.5 \mathrm{keV}$.

The observed system sensitivity for the digital systems is given by the slope of the plot on linear scale (the units are ADU/mR). From the slopes of Figure 2, we find the following values for the system sensitivity (left ordinate): $77 \mathrm{ADU} / \mathrm{mR}$

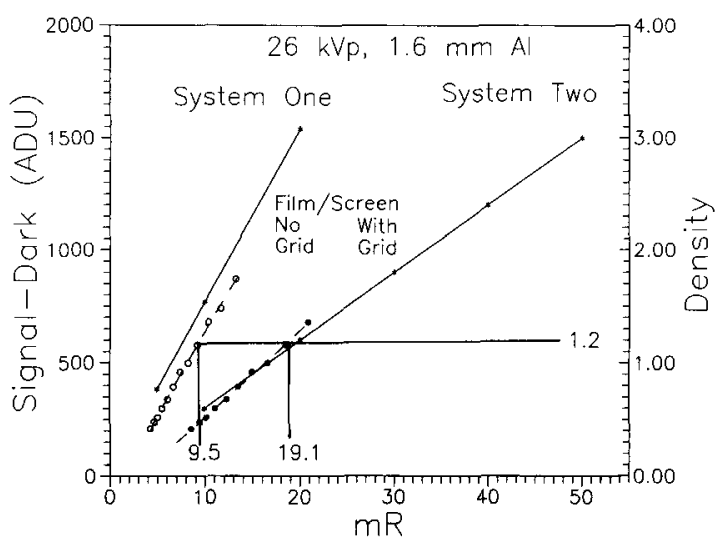

Fig 2. Sensitivity of system one and system two to $x$-ray exposure (solid graphs, left ordinate in units of ADU versus abscissa in milliroentgens) in comparison with the sensitivity of a film-screen system used with and without a scatter grid (dashed graphs, right ordinate in units of $O D$ versus abscissa in milliroentgens). for system one and $30 \mathrm{ADU} / \mathrm{mR}$ for system two. System one requires an exposure of about 27 $\mathrm{mR}$ to reach a response in the center of the dynamic range, ie, a response of 2,048 ADU, whereas system two requires an exposure of 68 $\mathrm{mR}$ to reach the same response of 2,048 ADU. Note also that the film-screen system requires an exposure of $9.5 \mathrm{mR}$ without the scatter grid to reach the optimum OD of 1.2 (right ordinate), whereas the exposure to reach the same OD with the grid is $19.1 \mathrm{mR}$. These values of optimum exposure for the film-screen combination are significantly smaller than the exposure of the digital systems to obtain a response in the center of their dynamic range. Clearly there is a problem for IQC, namely how to specify an optimal response for the digital systems.

These observations indicate that, contrary to the film-screen system, there is no apparent optimal exposure for digital imaging systems. At first glance, it might be reasonable to accept the response of a digital system as long as the response is somewhere within the dynamic range of the system, ie, larger than $\mathrm{ADU}, 0$, and smaller than $\mathrm{ADU}, 4,096$, for digital systems with 12 bits. On the other hand, the required exposures will cover a range of larger than 3 orders of magnitude, a range that could not be tolerated in IQC. Recall that the optimum response for the film-screen combination is OD, 1.2 , and exposures have to be repeated if the OD is significantly larger or smaller than 1.2 . Consequently, there is a definite need to define a measure of "optimum" and sufficient x-ray exposure. Fortunately, the quantitative nature of digital imaging systems permits finding that optimum exposure: Optimum and sufficient exposure should be based on the signal-to-noise ratio.

\section{Noise and Signal-to-Noise Ratio}

The measurement of noise in digital systems is similar to that with film: An aperture with area $A_{s}$ is scanned over the digital image and the $\mathrm{SD}$ about the mean digital value per pixel is derived using commonly used statistical procedures. In digital systems, this procedure can be easily accomplished because software is usually provided by the computers controlling the digital imaging systems. Unfortunately, the sampling aperture is fixed as a pixel with area $A_{p}$, ie, $A_{s}=A_{p}$. Rarely does one have the opportunity 
to vary the sampling aperture (which is relatively simple with film). However the measurement of mean and SD in larger apertures is also possible with digital systems, if one makes use of pixel binning. At the University of Arizona, such a program was written. This program forms sampling apertures larger than a single digital pixel in the form of binned pixels. Pixel binning is a common procedure in the CCD community where many pixels of a digital image are grouped together like four adjacent ones to form one large 2- $\times$ 2-pixel aperture, or nine adjacent ones to form a large 3- $\times 3$-pixel aperture. Now the sampling aperture consists of four pixels rather than one pixel $\left(A_{s}=4 A_{p}\right)$ or nine pixels rather than one pixel $\left(A_{s}=9 A_{p}\right)$. The program then finds the average value of the pixel values for the four, nine, or generally $n$ pixels forming the bin and provides the statistics about these averages. Figure 3 shows examples of noise as a function of the sampling aperture size (no. of pixels per bin), taken with systems one and two.

Note that, for both system one and system two, Selwyn's Law is not obeyed at small sampling apertures, ie, the variance is not inversely proportional to the area of the sampling aperture. ${ }^{18}$ It is hypothesized that Selwyn's Law can only be obeyed if the noise is white, ie, if the sampling aperture is larger than the imaging system's point spread function.

Figure 4 presents data on the maximum signal-to-noise ratio (for a contrast of $100 \%$ ) for flat-fielded images of system one and system two as a function of the $\mathrm{x}$-ray exposure in units

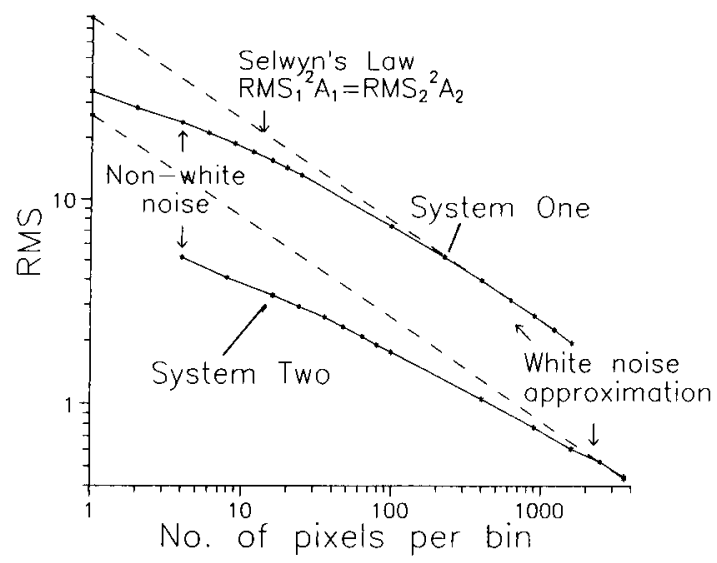

Fig 3. Noise (RMS) as a function of the sampling aperture area (in number of pixels per sampling aperture [generated in software by binning pixels]) for system one and system two.

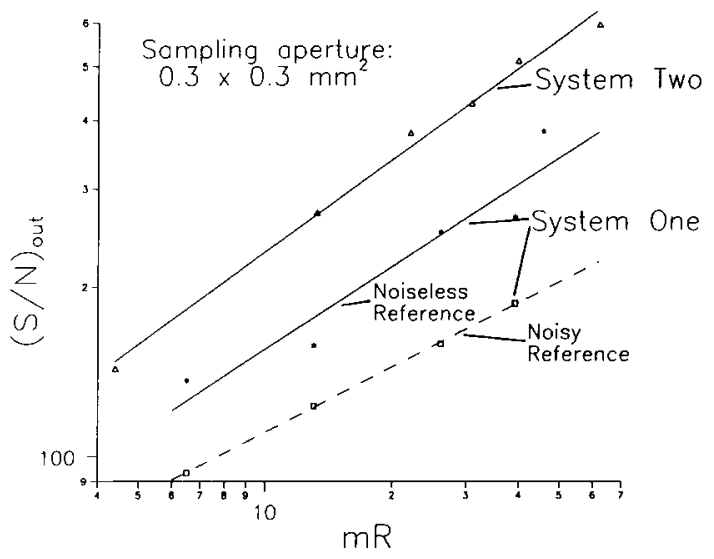

Fig 4. Output signal-to-noise ratio in a sampling aperture of size $0.3 \times 0.3 \mathrm{~mm}$ as a function of $x$-ray exposure for system one and system two.

of $\mathrm{mR}$ for a sampling aperture of linear dimension $0.3 \times 0.3 \mathrm{~mm}$. As is commonly done, signal-to-noise ratio is defined here as the ratio of the mean value in the aperture of $0.3 \times 0.3$ $\mathrm{mm}$ divided by the SD measured with the particular aperture size. The dashed line is based on images of system one that were collected when the reference image was noisy. As a result, the signal-to-noise ratio is very low.

This method of finding the signal-to-noise ratio in a given sampling area larger than the individual pixel size provides an objective measure of the performance of the imaging system that could easily be used not only for IQC, but also for acceptance tests. Of course, one has to make a decision on the diameter of the sampling aperture. Ideally it should match the size of an object of interest in mammography such as a microcalcification or a growing mass.

\section{Square-Wave Modulation of Different Spatial Frequencies}

Testing of the spatial resolution capabilities of imaging systems with the aid of limiting spatial resolution can only be a crude measure. It determines the highest spatial resolution an imaging system is capable of, but it does not show anything about how well spatial frequencies lower than the cut-off frequencies are imaged. A useful and practical alternative for IQC is the determination of the square-wave response at several spatial frequencies within the bandpass of the imaging system. This measurement is easily accomplished simply by imaging a commonly available lead-bar pattern. The square-wave response is obtained from the 
profile at several spatial frequencies, possibly by averaging over several adjacent image lines. The profile and the actual modulation are obtained by running a simple computer program. From the square-wave response, one can also obtain the sine-wave response with the aid of the Fourier Series, which is particularly easy if the frequencies of the square waves are selected as described by Hall. ${ }^{30}$

Figure 5 is an example for an experimental digital imaging system. It shows profiles of the image of a lead-bar pattern with spatial frequencies of $2,2.5$, and $3.15 \mathrm{lp} / \mathrm{mm}$. The measured values of square-wave modulation are $87 \%$, $82 \%$, and $75 \%$, respectively.

We mention in passing that the ideal measure of spatial resolution of an imaging system, the MTF, does not lend itself easily to an IQC program. The MTF is usually obtained from the Fourier Transform of the line-spread function, ${ }^{3,18}$ which takes a fair amount of time. Furthermore, it may not be necessary to determine the MTF every day.

\section{$X$-Ray Source Voltage}

The most accurate method to determine the voltage applied to the $\mathrm{x}$-ray source is to determine the $x$-ray spectrum and find the high energy cut-off of the spectrum. Recall that $\lambda_{\max }=(\mathrm{hc}) / \mathrm{kVp} .{ }^{12}$ However, such a measurement is not easily accomplished. ${ }^{13-16}$ An alternative method to find the applied voltage of the $x$-ray source is the inspection of images of an aluminum step wedge of say 10 steps, in a

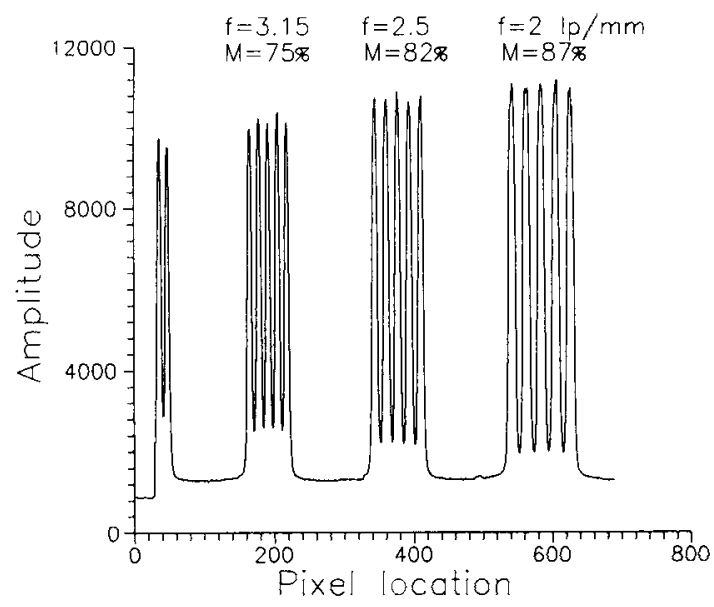

Fig 5. Square-wave response (modulation $M$ ) of an experimental $x$-ray imaging system at three spatial frequencies.
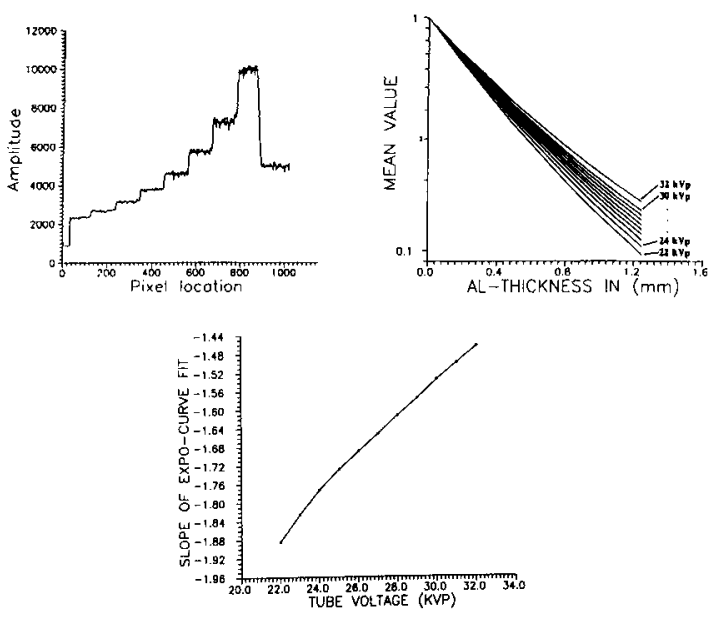

Fig 6. (A) Profile of the $x$-ray image of an aluminum step wedge when imaged with an experimental imaging system using a particular $x$-ray tube voltage. (B) Mean value of the steps of an aluminum stepwedge as function of aluminum thickness for different $x$-ray source voltages. (C) Shows slope of graphs of $B$ as function of the $x$-ray source voltage.

fashion similar to the determination of the effective energy in terms of HVL. ${ }^{12}$ The idea is to plot the digital value in each of the steps versus the thickness of the step in millimeters of aluminum and observe the exponent of a curve fit based on a power function. This exponent is a function of the applied voltage.

Figure 6 is an example for the case of a 10 -step aluminum step wedge with a step size of about $0.1 \mathrm{~mm}$ and a mammographic $\mathrm{x}$-ray source (molybdenum [Mo] target and Mo filter). The imaging system used was an experimental one, consisting of a CCD $(1,024 \times 1,024$ pixels $)$ with a straight fiber-optic window (magnification unity) and a phosphor screen, firmly pressed against the fiber optic.

Figure 6A shows one profile, Fig 6B shows the plot of the relative amplitude in each of the steps for $\mathrm{kVp}$ values from 22 to $32 \mathrm{kVp}$, and Fig $6 \mathrm{C}$ shows the plot of the slopes of the curves in Fig $6 \mathrm{~B}$ versus the applied voltage. Initial results indicate that this method permits estimation of the applied voltage to an accuracy of about $5 \%$.

\section{$X$-Ray Spectrometer Based on Crystal Diffraction Spectrometry}

The estimation of performance characteristics such as detective quantum efficiency (DQE) for $\mathrm{x}$-ray imaging systems requires frequently a precise knowledge of the $\mathrm{x}$-ray spectrum incident on the image detector. ${ }^{18,31}$ The usual ab- 
sence of adequate instrumentation like a germanium spectrometer ${ }^{13-16}$ requires retreat to other much cruder methods to estimate the energy characteristics of the $\mathrm{x}$-ray beam, such as the HVL in millimeters of aluminum. However, such estimation is very crude and can cause large errors.

Recently a simple device was described that provides an $\mathrm{X}$-ray spectrum based on wavelength dispersive crystal diffraction spectrometry ${ }^{32}$ Figure 7A shows the device schematically. The original schematic was made to describe the system with film as the read-out detector (recorder). However, removal of this film and replacing it with a digital imaging detector such as the combination of phosphor-fiberoptic taper $\mathrm{CCD}$ will turn the device into a real time $\mathrm{x}$-ray spectrometer. In fact, placing the device without film in between the x-ray source (the spectrum of which is to be evaluated) and the image detector of a digital imaging system, such as system one or system two, permits determining the spectrum of the $\mathrm{x}$-ray source of those digital systems in real time. Of course, calibration is necessary with respect to the location of the device between the $x$-ray focal spot and the image detector, as well as with respect to the energy sensitivity of the digital image detector. It appears that the device is simple enough that it can be used in IQC, maybe not for every day, but certainly once a month.

Figure 7B shows spectra of a mammographic $\mathrm{X}$-ray source at various $\mathrm{x}$-ray source voltages taken with a film-based device. Note the characteristic lines of Mo (from the Mo-anode). The particular beauty of such a real time x-ray spectrometer is that a useful spectrum can be obtained during the time of a full x-ray exposure, whereas it takes considerable time to determine the $\mathrm{x}$-ray spectrum using pulseheight distributions in semiconductor detectors such as a germanium detector. ${ }^{13-16}$

\section{X-Ray Focal-Spot Size}

The size and the shape of the x-ray focal spot of an $\mathrm{x}$-ray source is usually obtained with the aid of a pinhole and a film-screen combination. The pinhole is placed as close as possible to the $x$-ray source's focal spot and an exposure is made. If the pinhole is aligned properly along the central beam from the focal spot to the
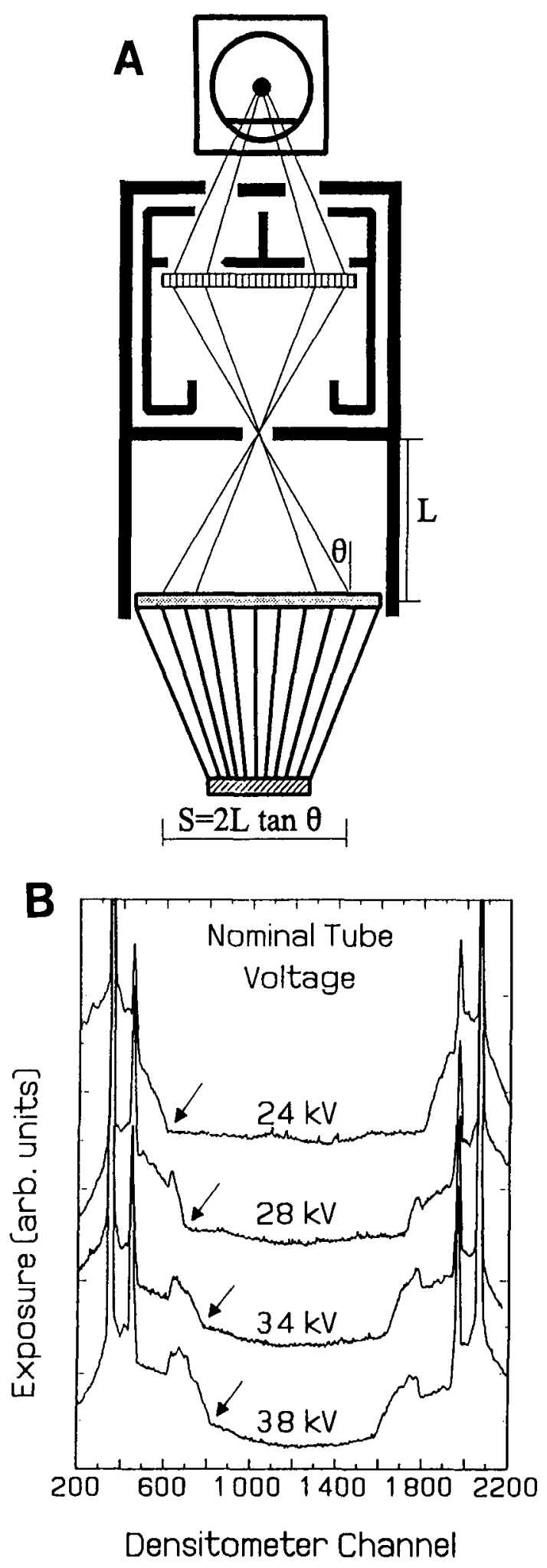

Fig 7. (A) Schematic of digital $x$-ray spectrometer based on crystal diffraction. (B) $X$-ray spectra measured with crystal diffraction-based $x$-ray spectrometer when film was used as image detector (reprinted with permission from Deslattes et al. ${ }^{32}$. 
center of the imaging detector, a pinhole image of the focal spot will be projected onto the image detector, and one can observe the image of the focal spot on the developed film.

This analog pinhole camera can be turned into a digital camera by replacing the filmscreen combination with a digital system such as a CCD camera with a screen coupled fiberoptically to the CCD. The focal spot image would be presented on a CRT and the focal spot size can be obtained with the aid of a cursor in units of numbers of image pixels. Of course, the actual size of the focal-spot image on the sensor of the digital $\mathrm{x}$-ray camera is obtained by multiplying the number of image pixels subtending the focal-spot image with the pixel size in millimeters. Correction for the pinhole magnification and the angle of the $x$-ray source's anode will result in the actual focal-spot size. Specifically for the case of digital mammography cameras, one can obtain focal-spot images of the $\mathrm{x}$-ray source, which is part of the digital imaging device directly and in real time. This is achieved simply by placing the pinhole as close as possible to the collimator, well aligned within the center of the X-ray beam from the focal spot to the center of the digital imaging system. In fact, one can place the pinhole into different positions within the field of view of the imaging system, specifically to obtain the effective focalspot size close to the chest wall. Figure 8 is an example of a focal-spot image obtained from system two. Again, the procedure is simple enough that it can be included in IQC.

\section{Use of Phantoms}

Of particular interest with respect to IQC was the performance of the digital systems when images of phantoms such as the ACR accreditation phantom and the CDMAM phantom were involved. Unfortunately, the field of view of the imaging systems available was only $5 \times 5 \mathrm{~cm}$, whereas the size of the ACR accreditation phantom is about $10 \times 10 \mathrm{~cm}$ and that of the CDMAM phantom is about $20 \times 25 \mathrm{~cm}$. Therefore, it was never possible to generate a phantom image with the digital systems that contained all phantom objects simultaneously.

ACR-accreditation phantom. Images were generated at various $x$-ray energies and exposure levels. Unfortunately, we did not observe

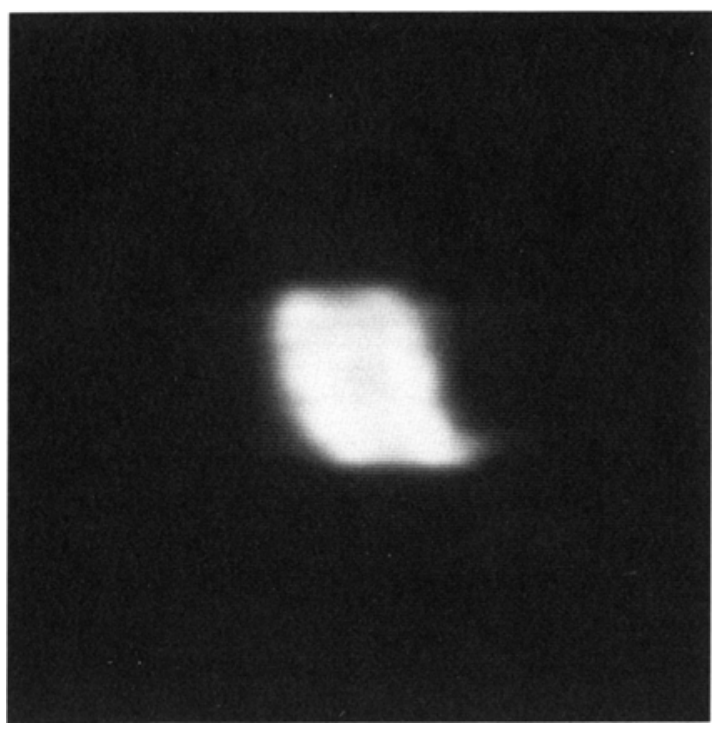

Fig 8. Image of focal spot of $x$-ray source used with system two and imaged with system two with the aid of a $30-\mu \mathrm{m}$ pinhole.

systematic changes as various energies and exposures were used. This may have been caused by the fact that the phantom is not really a good phantom for measurement of image quality as explored through contrast-detail studies. The display signal-to-noise ratio or the contrasts may have been always high enough such that detection was $100 \%$. Figure 9A shows an image of a portion of the ACR accreditation phantom (the four thinnest fibrils are included), taken with system two. Figure 9B shows the same portion of the ACR phantom, but taken with system one. Notice that the image of system one is much noisier than that of system two. Notice also that the image provided by system one is more nonuniform than that of system two, such that the smallest fibril can barely be detected.

In general, the digital imaging systems performed significantly better than the conventional film-screen systems in that more objects were seen. With system one, 14 of the total of 16 objects could be detected: the smallest mass and the smallest calcification cluster could not be seen. With system two, 15 of the total of 16 objects could be detected. Only the smallest set of calcifications could not be seen.

CDMAM phantom. The CDMAM contrastdetail phantom was used with both system one and system two in a systematic contrast-detail study. ${ }^{33,34}$ Figure 10, A (above) and B (below) 

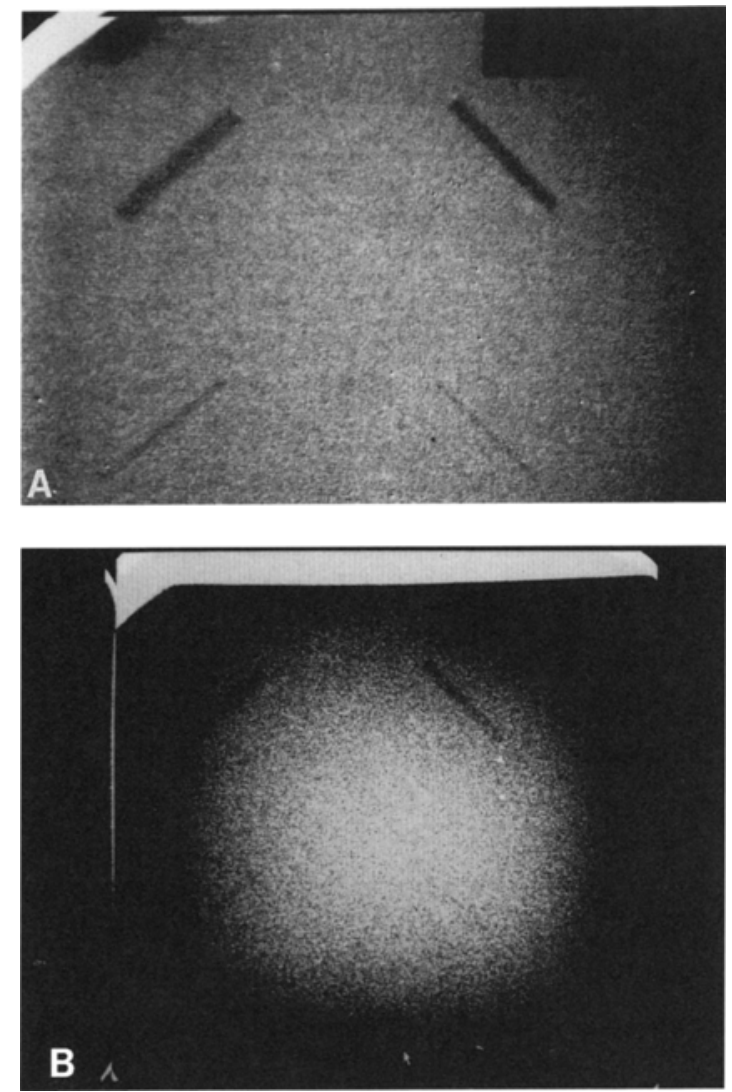

Fig 9. Portion of ACR accreditation phantom imaged with system two (A) and system one (B).

show images of a particular section of the phantom taken with system two and system one, respectively, at the exact same exposure setting ( 2 plates, $27 \mathrm{kV}, 500 \mathrm{mR}$ ). Notice that, just like in the case of the ACR Accreditation phantom, the images provided by system one are noisier than those of system two. More noise indicates a lower signal-to-noise ratio for system one, possibly caused by a lower DQE than that of system two. This assumption is in agreement with the physical measurements reported elsewhere. ${ }^{3}$ Notice again that the image for system one is much more nonuniform than that of system two. This nonuniformity makes it more difficult to enhance the display contrast evenly throughout the field. As a result, faint objects cannot be detected, even though the signal-to-noise ratio would be sufficient for detection.

The results of the contrast-detail experiment are plotted in Fig 11 in terms of threshold thickness in units of micrometers (equivalent to "threshold contrast") at $62 \%$ probability of detection as function of object diameter. The data pertaining to system one are marked as "F", those pertaining to system two are marked as " $L$ ". The phantom was used with four plexiglass plates, and two x-ray source voltages were used: $27 \mathrm{kVp}$ and $30 \mathrm{kVp}$. Clearly, system two provides detection of a significantly lower object thickness (corresponding to a lower thresholdcontrast) than system one. This result indicates a higher signal-to-noise ratio or better DQE for system two in close correlation with the measurements on DQE reported elsewhere. ${ }^{3}$

It is clear that the use of a contrast-detail phantom such as the CDMAM-phantom in IQC can provide quantitative information on the signal-to-noise ratio of digital imaging systems. Detection of an object with a particular diameter and thickness would indicate a particular
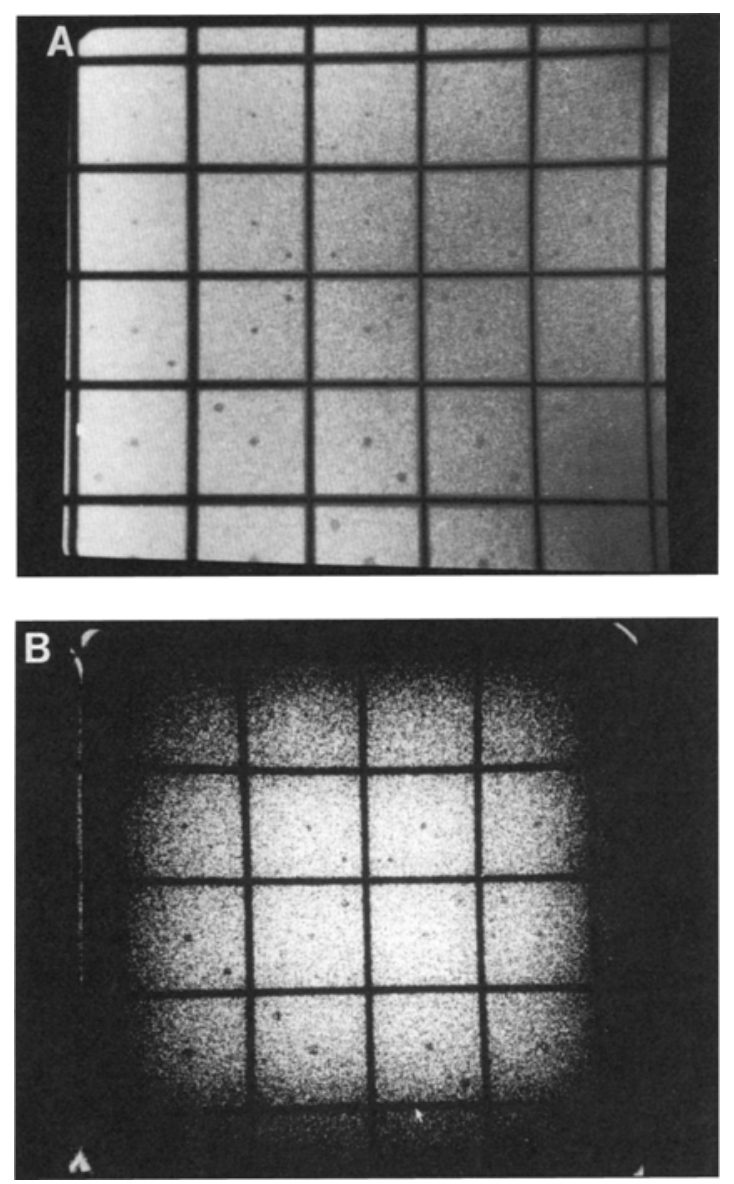

Fig 10. Portion of CDMAM phantom, imaged with system two using two plates, $27 \mathrm{kVp}$, and $500 \mathrm{mR}(\mathrm{A})$, and same portion of CDMAM phantom, imaged with system one under the same conditions (B). 

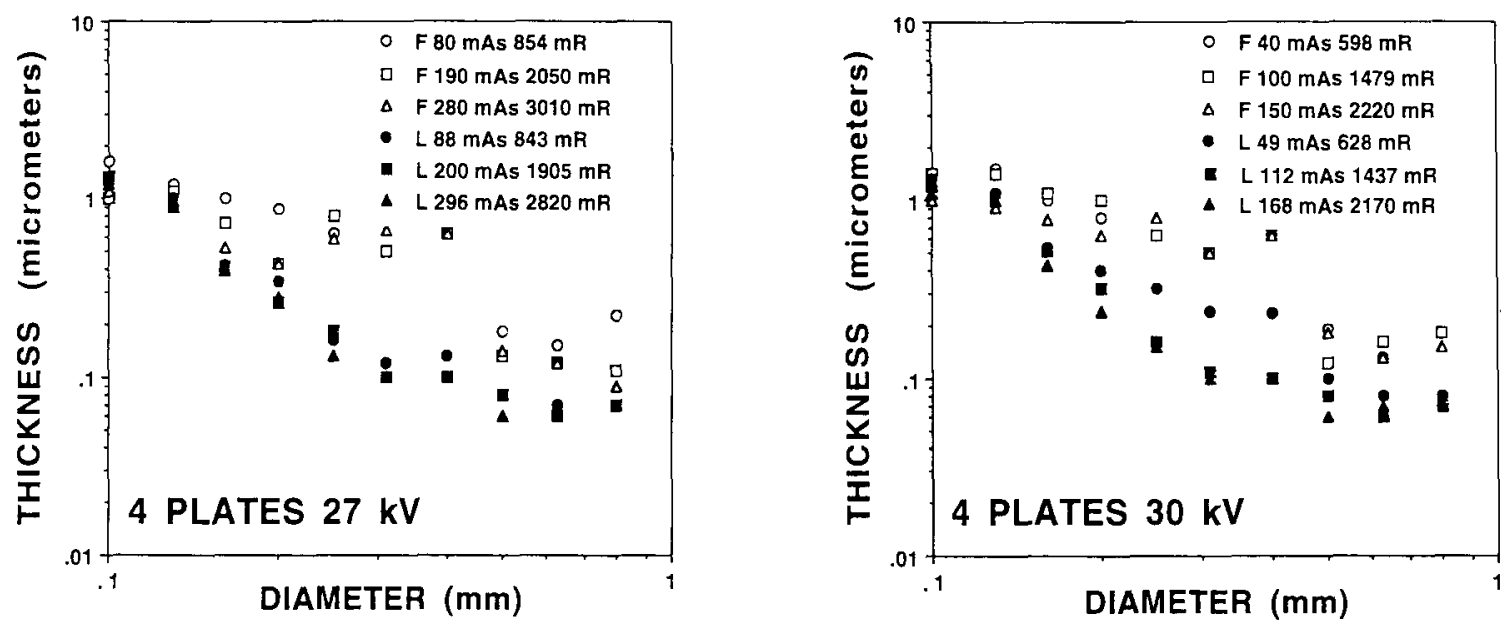

Fig 11. Threshold thickness of discs in CDMAM phantom, detected correctly with $62 \%$ probability of detection (threshold contrast) as function of disc diameter for all exposure conditions, using system one (curves labeled " $F$ ") and system two (curves labeled "L").

signal-to-noise ratio, which was achieved with a particular x-ray fluence with a given effective energy. Assuming that $\mathrm{x}$-ray fluence and effective energy had not been changed, proper system operation could be related to repeated detection of the particular phantom object.

\section{Cathode-Ray Tube Display (CRT)}

Displaying images on CRTs generates an additional problem that will have to be addressed sooner or later in IQC, namely that of standardization. From the beginning of digital medical imaging to this day, the radiologic community has been plagued by two imagepresentation problems. The problems are encountered when presenting electronically acquired digital images on either soft copy, such as CRT monitors, or hard copy (films). First, when an image is displayed on a CRT, the gray scale is not necessarily reproducible from monitor to monitor and over time. Even if the presentation is with a film-like gradation curve, the soft copy gray-scale rendition is not automatically the same as seen on film. Secondly, when transferring an image with gray scale satisfactorily presented on the CRT display of a workstation to another soft copy display, the rendition of gray scale on the second monitor is again not automatically identical to the first one.

These problems have contributed to reservations held by many radiologists about working with soft copies. These problems exist largely because softcopy display systems, as well as many electronic image printers, do not render gray scale according to a standard, rather they can produce an almost infinitely large number of gray scale renditions. The basic advantage of the digital imaging system, the separation of the functions of detection and display, turns out to be a burden in the sense that images will rarely look alike unless one agrees on a specific display function for all displays, ie, a standard. Over and above, one should agree upon an optimum display function that maximizes the information transfer to the human observer. A standard has been proposed many times ${ }^{35-37}$ that is based on perceptual linearization. Perceptual linearization produces a gray scale in which equal changes in driving signals (command levels, digital input values) produce changes in luminance that are perceptually equivalent throughout the entire luminance range. Only when using a standardized gray scale rendition will quantitative observer performance measurements with more general meaning be performed on electronic image displays.

Unfortunately, this subject has not been settled yet. In particular, it appears that perfect perceptual linearization cannot be generated until display buffers are available which offer digital-to-analog converters with more than 8 bits. ${ }^{37}$ Therefore, the suggestion is made to perform IQC for CRTs with the aid of the Society of Motion Pictures and Television Engineers (SMPTE) test pattern. This pattern is available as a software package to be stored in 
the display controller of the computer. ${ }^{38,39,40}$ The pattern consists of 10 gray steps, several high- and low-contrast resolution bar patterns, and two contrast steps of about $5 \%$, one at the low end of the dynamic range (dark) and one at the high end (bright). The contrast-brightness controls of the monitor are set such that the two contrast steps are visible. Subsequently, the contrast-brightness controls should never be touched. The high resolution and the lowresolution bar patterns serve to test spatial resolution and the CRT's spatial noise (phosphor granularity), respectively. IQC of the monitor consists of displaying the SMPTE test pattern to verify proper setting of the contrast brightness controls, and to check visibility of the bar patterns. In addition, quantitative measures can be taken, namely the maximum luminance in the $100 \%$ signal field of the pattern, the dynamic range as defined by the ratio of maximum to minimum luminance and the display function, which is a curve plotting the luminance versus the digital input level. ${ }^{41}$

Figure 12 shows the display curves for the CRTs of both digital systems, based on adjustment of the CRT's contrast-brightness controls with the aid of the SMPTE pattern. The maximum luminance for the CRT of system one is $\mathbf{5 7}$ foot-lamberts, whereas the maximum luminance for the CRT of system two is about 50

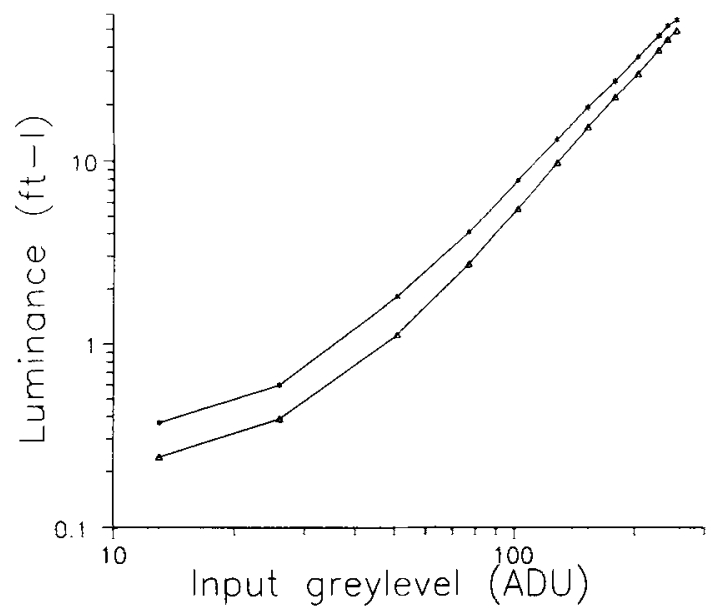

Fig 12. Monitor luminance as function of input signal (in digital units) for CRT monitors used with system one $(")$ and system two $(\triangle)$. For the measurement, the contrast-brightness controls were set to a position, where the $0 \%$ to $5 \%$ and $95 \%$ to $100 \%$ contrast objects of the SMPTE pattern were equally visible. The luminance was measured in the 10 steps of the SMPTE pattern. foot-lamberts. The dynamic ranges for the CRTs of system one and system two are 176 and 260 , respectively. It is to be noted here that this maximum luminance of about 60 foot-lamberts is almost an order of magnitude smaller than the maximum luminance of a typical film light box, which is about 500 foot-lamberts. ${ }^{35}$

\section{VARIATIONS OF PERFORMANCE PARAMETERS AS A FUNCTION OF TIME}

During a period of approximately 1 year, we were able to observe a variety of performance parameters. The following is an assembly of examples. These examples may not be typical for any particular system, they merely show that a variety of actually simple problems can occur in a digital imaging system.

In general, for both digital systems, mean and SD were very stable for the same $x$-ray exposure settings, most often to within a single digital unit. This high stability indicates that both the camera systems and the x-ray generators and $x$-ray sources are very stable. However, every so often there were drastic changes, indicating that something had been changed or that something must have gone wrong. The following few examples show that Murphy's law is very much valid: If something can go wrong, it will go wrong! Figure 13, A and B show schematic examples for mean and $\mathrm{SD}$ at a particular exposure level (not necessarily the same) for both digital systems as a function of time. Changes of mean and SD (indicating changes in sensitivity) were caused by the following:

Over-exposure when generating the reference image. This condition caused saturation of the reference image. If the reference image is overexposed, and effectively consists of a constant, the flat-fielding process will be ineffective, ie, it will not remove the fixed pattern noise from the raw image, which is exactly what happened in those incidents.

Scatter grid left in field. System one has a provision to perform examinations with a filmscreen combination. The switch from digital to film-screen is accomplished simply by placing a stationary scatter grid with an associated filmscreen holder in front of the digital camera. Also the manufacturer of system one encouraged users to take a reference image (necessary for the flat-fielding process) as often as possible, 

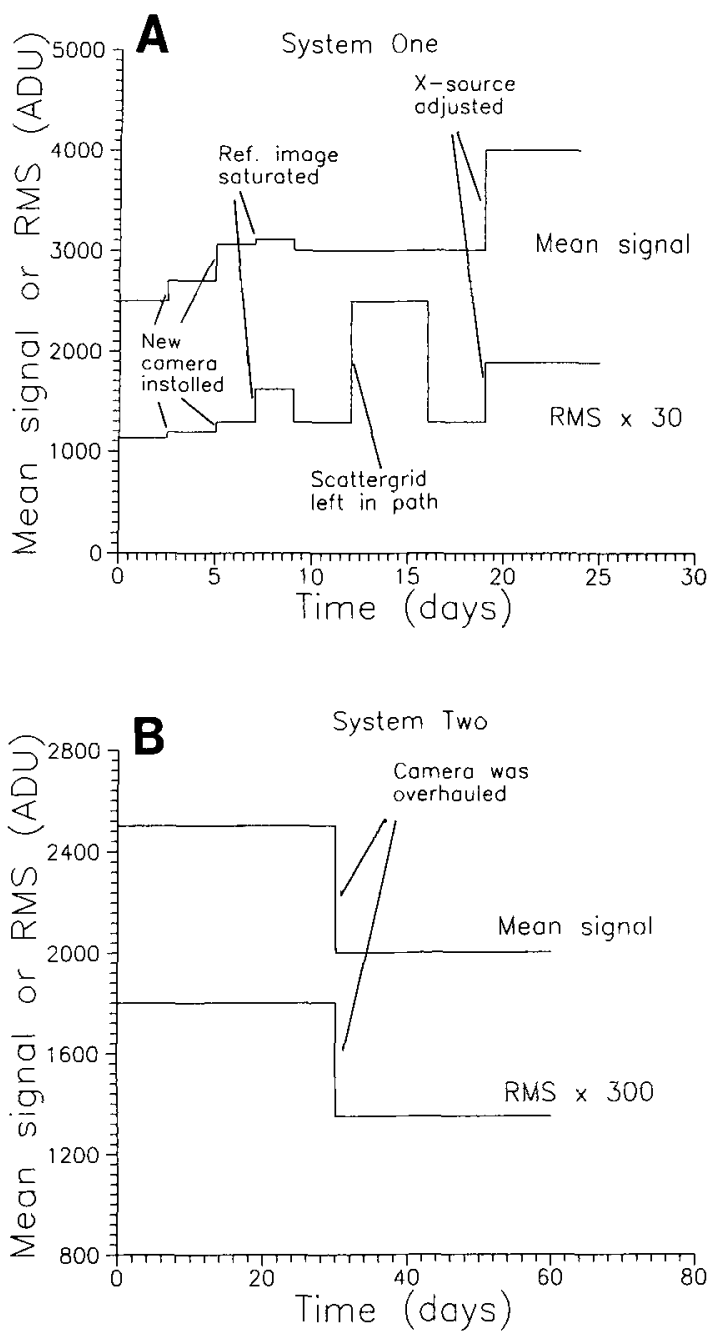

Fig 13. (A) Schematic change of mean and SD of system one as function of time. (B) Schematic change of mean and SD of system two as function of time.

even every day, before beginning procedures. Unfortunately, every so often after the filmscreen combination had been removed, the scatter grid was left in place in front of the digital camera for some time. During this time, the digital system was operated and the reference image was taken with the scatter grid in place. Therefore, the grid lines would be present in both the raw and the reference image. A major part of the gridlines would disappear in the flat-fielding process, except one would notice Moire-patterns, because of small misalignment of the grid lines with respect to the CCD raster lines. Sooner or later, the technologists would notice the presence of the scatter grid and remove it. However no new reference image was taken, which means the reference image with the gridlines would continue to be used for flat fielding. As a result, all flat-fielded images would show the grid lines, causing an increase in the RMS.

Adjustments made by the company service personnel to the gain settings of the camera or to the voltage and current controls of the x-ray generator. The manufacturer of system one replaced the CCD camera three times, resulting in three different sensitivities. In addition, they adjusted either the current calibration or the $\mathrm{kVp}$ calibration in the x-ray source, resulting in a different $\mathrm{x}$-ray output in terms of milliroentgens/milliamperes. The manufacturer of system two adjusted the CCD camera once, resulting in a decrease in the sensitivity.

Another problem observed, which clearly affects IQC, particularly with respect to the CRT, was the burn-in of the company logo into the phosphor screen of the CRT. During the whole time that system one was at the University of Arizona, the system power was never turned off, and the system was always ready for use, including the CRT monitor. The only provision with respect to the monitor was to turn the beam off by setting the contrast-brightness controls to their minimum positions. Unfortunately this procedure did not completely turn off the CRT's scanning beam, so the company Logo was slowly imprinted into the CRT phosphor as shown in Fig 14. Such a pattern on a CRT will interfere

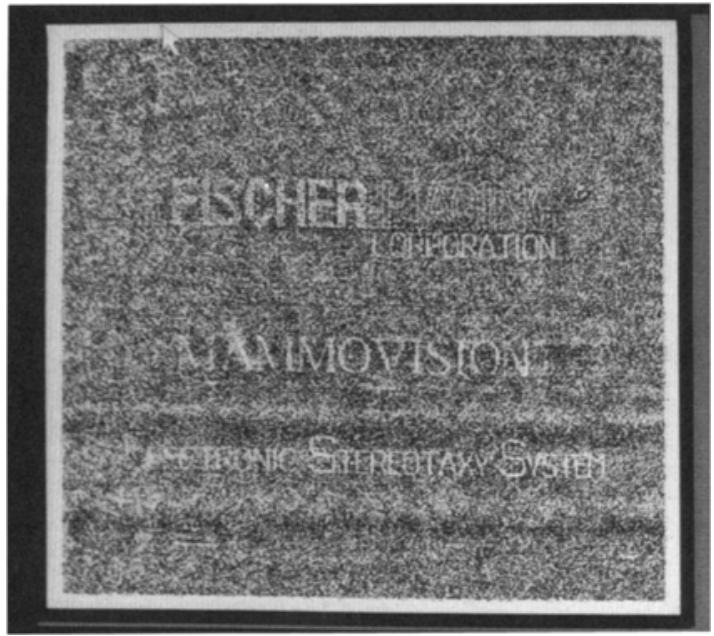

Fig 14. The company logo burned slowly but surely into the phosphor of CRT for system one because of the absence of a screen saver. 
with detection of subtle contrasts in the anatomy of interest. It could be avoided by use of a screen-saver.

\section{CONCLUSION}

IQC procedures for digital mammographic imaging systems can take advantage of real time and digital capabilities of the system and provide many more quantitative results than possible with IQC for the conventional film-screen combination: monitoring mean and $\mathrm{SD}$ in a given region of interest at a known $\mathrm{x}$-ray exposure as a daily test will find major changes in system parameters; the signal-to-noise ratio can be determined using a sampling aperture larger than a pixel (pixel binning), where the size of the sampling aperture has to be specified; the square-wave modulation can be measured at certain spatial frequencies; the measurement of $\mathrm{x}$-ray focal spot of the digital mammography system can be accomplished by projecting a pinhole image of the focal spot onto the digital image detector of the x-ray imaging system; the $x$-ray source voltage can be obtained from images of an aluminum stepwedge; the measurement of the $x$-ray spectrum of the $x$-ray source may be easily accomplished with a spectrometer based on crystal diffraction; the CRT of the digital imaging systems needs to be included in IQC using at least an SMPTE pattern for monitoring of maximum luminance, dynamic range and spatial resolution; the use of screen savers will prevent unnecessary burn-in of patterns like the manufacturer's logo into the screen of the CRT; the CDMAM contrastdetail phantom is an ideal phantom for contrastdetail measurements and can be used in comparisons of image quality for different imaging systems; and the ACR Accreditation phantom may need to be redesigned because currently used digital imaging systems are very close to detecting all objects.

It is time to develop standards for image quality of digital imaging systems for use in mammographic applications.

\section{ACKNOWLEDGMENT}

We thank Emre Toker from the Fischer Imaging Corporation and Milt Stoller from LoRad Corporation for their help in the analysis and operation of the cameras and Hy Glasser and Martin J. Ratner of Nuclear Associates for providing the CDMAM phantom for the study.

\section{REFERENCES}

1. Gray JE, Winkler NT, Stears J, et al: Quality Control in Diagnostic Imaging. Baltimore, MD, University Park, 1983

2. Roehrig H, Fajardo L, Yu T: Digital x-ray cameras for realtime stereotactic breast needle biopsy. SPIE 1896:213224, 1993

3. Roehrig H, Schempp WS, Fajardo L, et al: Signal, noise and detective quantum efficiency in CCD based $x$-ray imaging systems for use in mammography. SPIE 2163:320332,1994

4. Haus AG, Yaffe MJ (eds): Syllabus: A Categorical Course in Physics: Technical Aspects of Breast Imaging (2nd ed). Oak Brook, IL, RSNA Publications, 1993

5. Maidment ADA, Yaffe MJ, Plewes DB, et al: Imaging performance of a prototype scanned-slot digital mammography system. SPIE 1896:93-103, 1993

6. DeParedes ES, Frazier AB, Hartwell GD, et al: Development and implementation of a quality assurance program for mammography. Radiology 163:83-85, 1987

7. Hendrick RE: Standardization of image quality and radiation dose in mammography. Radiology 174:648-654, 1990

8. McLelland R, Hendrick RE, Zinninger MD, et al: The American College of Radiology Mammography Accreditation Program. AJR 157:473-479, 1991

9. Kimme-Smith C, Bassett L, Gold RH, et al: Mammography screen-film selection: Individual facility testing techniques. Med Phys 19:1195-1199, 1992
10. Kimme-Smith C, Bassett L, Gold RH, Fox SA, Solberg T: Testing mammography equipment: Evolution over a 4-year period. Med Phys 19:1491-1494, 1992

11. FDA's Plans for Implementing MQSA '92: Radiol Imaging Letter 13:137-139, 1993. (published by Pacela AF, 1351 Titan Way, Brea, CA 92621)

12. Johns HE, Cunningham JR: The Physics of Radiology (3rd ed). Springfield, IL, Thomas, 1974

13. Fewell TR, Weaver KE: The measurement of diagnostic $\mathrm{x}$-ray spectra with a high purity Germanium spectrometer. SPIE 56:9-18, 1975

14. Seltzer SM: Calculated response of intrinsic Germanium detectors to narrow beams of photons with energies up to $300 \mathrm{keV}$. Nucl Instr Methods 188:133-151, 1981

15. Seelentag WW, Panzer W: Stripping of $x$-ray bremsstrahlung spectra up to $300 \mathrm{kVp}$ on a desk type computer. Phys Med Biol 24:767-780, 1979

16. Laitano RF, Pani R, Pellegrini RR: Determination of $x$-ray spectra and of the scattered component up to $300 \mathrm{kV}$. Med Phys 18:934-938, 1991

17. Law J: The measurement and routine checking of mammography $x$-ray tube kV. Phys Med Biol 36:1133-1139, 1991

18. Dainty JC, Shaw R: Image science: Principles, analysis and evaluation of photographic-type imaging processes. San Diego, CA, Academic, 1974

19. Nishikawa RM, Yaffe MJ: Signal-to-noise properties 
of mammographic film-screen systems. Med Phys 12:32-39, 1985

20. Rose A: Vision: Human and Electronic. New York, NY, Plenum, 1973

21. Rosell F, Willson A: Recent psychophysical experiments and the displayed signal-to-noise ratio concept, in Biberman LM (ed): Perception of Displayed Information. New York, NY, Plenum, 1971, pp 167-232

22. Cohen G: Contrast-detail analysis of imaging systems: Caveats and kudos, in Doi K, Lanzl L, Lin PJ (eds): Recent Developments in Digital Imaging. Medical Physics Monograph 12:141-159, 1985

23. Karssemeijer N, Frieling JTM, Hendricks JHCL: Spatial resolution in digital mammography. Invest Radiol 28:413-419, 1993

24. Capp MP, Roehrig H, Seeley GW: The digital radiology department of the future. Radiol Clin N Am 23:349-356, 1985

25. Dallas WJ, Ovitt TW, Capp MP, et al: A prototype totally digital radiology department: Conception and initiation. SPIE 767:700-707, 1987

26. Seeley GW, Ovitt TW, Capp MP: The total digital radiology department: An alternative view. AJR 144:421, 1985

27. Dallas WJ: A digital prescription for $\mathrm{x}$-ray overload. IEEE Spectrum 27:33-46, 1990

28. Charge Coupled Devices for Quantitative Electronic Imaging. Revision H. Tucson, AZ, Photometrics Limited, 1992

29. Fahrig R, Maidment ADA, Yaffe MJ: Optimization of peak kilovoltage and spectral shape for digital mammography. SPIE 1651, 1992

30. Hall JA: Evaluation of signal-generating image tubes, in Biberman LM, Nudelman S (eds): Photoelectronic Imaging Devices, vol 2. New York, NY, Plenum, 1971, pp 77-115

31. Hillen W, Schiebel U, Zaengel T: Imaging perfor- mance of a digital storage phosphor system. Med Phys 14:744-751, 1987

32. Deslattes RD, Levin JC, Walker MD, et al: Noninvasive high-voltage measurement in mammography by crystal diffraction spectrometry. Med Phys 21:123-126, 1994

33. Krupinski EA, Roehrig H: Comparison of digital $\mathrm{x}$-ray systems for stereotactic breast needle biopsy: An observer performance study. Presented at the Second International Workshop on Digital Mammography, York, UK, July 10-12, 1994

34. Krupinski EA, Roehrig H, Yu T: Observer performance comparison of digital $\mathrm{x}$-ray systems for stereotactic breast needle biopsy. Acad Radiol 2:116-122, 1995

35. Blume $\mathbf{H}$, Roehrig $\mathrm{H}$, Browne $\mathrm{M}$, et al: Comparison of the physical performance of high resolution CRT displays and films recorded by laser image printers and displayed on light-boxes and the need for a display standard. SPIE 1232:97-114, 1990

36. Blume H, Daly S, Muka E: Presentation of medical images on CRT displays: A renewed proposal for a display standard. SPIE 1897:215-231, 1993

37. Hemminger BM, Johnston RE, Rolland JP, Muller KE: Perceptual linearization of video display monitors for medical image presentation. SPIE 2164:224-240, 1994

38. Lisk KG: SMPTE test pattern for certification of medical diagnostic display devices. SPIE 486:79-82, 1984

39. Gray JE, Lisk KG, Haddick DH, et al: Test pattern for video displays and hard-copy cameras. Radiology 154:519527,1985

40. Gray JE, Anderson WF, Shaw CC, et al: Multiformat video and laser cameras: History, design considerations, acceptance testing, and quality control: Report of AAPM Diagnostic X-Ray Imaging Committee task group no. 1. Med Phys 20:427-438, 1993

41. Roehrig H, Blume H, Ji TL, et al: Performance tests and quality control of cathode ray tube displays. J Digit Imaging 3:134-145, 1990 